\title{
Node Position Effect on Link Reliability for Body Centric Wireless Network Running Applications
}

\author{
Haider A. Sabti \& David V. Thiel \\ Griffith School of Engineering, Griffith University \\ Brisbane, QLD Australia \\ haider.al-husseinawi@griffithuni.edu.au,d.thiel@griffith.edu.au
}

\begin{abstract}
Health and sport applications have been a flourishing area for deploying Wireless Body Area Networks (WBAN) as this technology can provide a real time feedback which is important for the user, coaches, doctors and the viewing public. A wireless accelerometer sensor module was used to determine the link performance by recording the data and traffic lost on different runners and for different transmitter locations around the human body (foot, leg and arm). An approximate swing time calculation algorithm was employed to find the swing time effect on these losses. Experimental measurements showed $98 \%$ reliability at $250 \mathrm{kbps}$ while $62 \%$ resulted when using a data rate of $2 \mathrm{Mbps}$. The results also showed that the sensor on the wrist gives the best outcome from the locations tested.
\end{abstract}

Keywords-WBAN; WPAN; Body Centric Wireless Network; On Body Communication; Wireless Link Reliability; Sport; Running; Accelerometer; Swing time

\section{INTRODUCTION}

G AIT ANALYSIS requires the capture of body movement actions such as walking or running. The most common methods of video recording and subsequent image processing require complex analysis algorithms [1]. The new concept for capturing human movements utilize Wireless Body Area Network (WBAN) with movement sensors. The ongoing efforts on the miniaturization of hardware embedded devices and sensors have made on-body communications more practical during human movement using these wearable and smart wireless sensors [2].

The growing adoption of Wireless Body Area Networks (WBAN) has led to improvements in this technology through antenna design and communications strategies. Sensors implanted in or on the human body are being rapidly adopted in health and sports monitoring applications [3]. The variety of commercially available sensors such as accelerometer and gyroscope, electrocardiography (ECG), electromyography (EMG), and electroencephalography(EEG), carbon dioxide $\left(\mathrm{CO}_{2}\right)$, blood pressure, temperature sensors have encouraged the use of WBAN in e-health and e-sport projects [4]. The objectives include identifying the human gesture recognition, movement detection and body vital signs monitoring and sending this information to a central node for local monitoring and processing.
The wireless network performance is driven by its ability to successfully handle traffic and is affected by many parameters such as operating frequency, transmitted power, receiver sensitivity, channel data rate and the distance between the wireless nodes [5]. Unfortunately the propagation of the radio waves is affected by the presence of the human body as an obstacle [6]. In running, the swing time is defined as the time between each consecutive foot contacts with the ground of the right or left leg [7]. During the natural human swing action the body limbs can interfere with the line of sight (LOS) propagation path between the transmitting and receiving nodes resulting in multipath fading and scattering effect of the transmitted signals which affect the network reliability.

The WBAN usually involves the use of small, smart wireless nodes located on or in the human body and connected to a local network to provide both ON body and OFF body communications. In this way real-time feedback can be provided to the user or professional personnel [8]. This paper reports the analysis of the wireless link performance in body area networks and follows previous work on WBAN near real time feedback using movement based time division multiplexing [9]; in which a simple version of the time shift error correction algorithm was proposed to correct the time error difference between transmissions [10].

\section{MEASUREMENT SYSTEM AND PROCEDURE}

\section{A. System}

The system consists of a wireless master/slave transceiver sensor module, the slave node programmed to log only the data received from the master node and to log nothing when there is no transmission (transmission stop or data lost). Both wireless nodes have the same hardware structure but they were programmed with different code to perform their tasks in C language with Atmel Studio 6.1 to implement the codes. Each node consisted of a MCU (ATmega324P Microcontroller), wireless transceiver chip module (NRF24L01+) with a built-in PCB meander-line monopole antenna, accelerometer sensor (MMA7260QT), memory (AT45DB161D) and a power source (as shown in Fig. 1) in the form of $3.6 \mathrm{~V}$ battery with a maximum capacity of $240 \mathrm{~mA}$. The node life time can be extended for around three hours, which is sufficient to monitor a complete training session. 


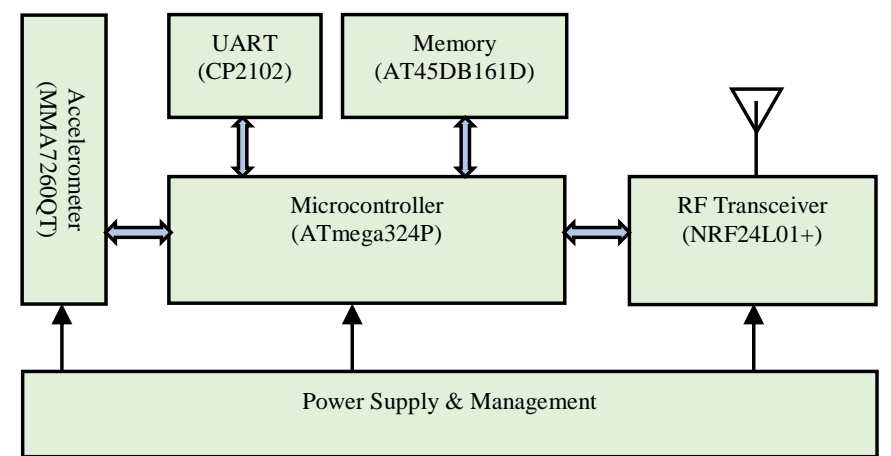

Fig. 1. Hardware structure of the wireless sensor node.

Each node has a UART interface (CP2102) which allows wired data transfers to a local computer for real-time monitoring or further processing during or after the completion of the sport activity. The accelerometer sensor measures the analog acceleration data of any movement during the gait cycle which is converted by the MCU to a digital form of $(\mathrm{mV} / \mathrm{g})$. These data are recorded in the memory and transmitted or received using the wireless transceiver.

\section{B. Procedure}

The test involved the participation of human volunteers, who were asked to wear the wireless transmitter and receiver nodes and then run in a sports field; an outdoor environment was chosen to minimize possible multipath effects from nearby objects. The volunteers were fully informed about all relevant information of the project as per the code for responsible conduct of research (Ethic approval ENG/20/13/HREC). The nodes were configured to continually transmit and receive data at the same power level and frequency [0 $\mathrm{dBm}$ and $2.4 \mathrm{GHz}]$ and data rates of $250 \mathrm{kbps}, 1 \mathrm{Mbps}$ and $2 \mathrm{Mbps}$, respectively. The running data of five runners of different styles and abilities were analyzed to assess variability. The wearable nodes were attached in fabric bands to ensure stability during movement and for easy on body mounting. The transmitting node was placed at six different locations to the outside part of the wrist and arm (above the elbow), the upper surface of the foot, the lower and upper parts of the leg and on the thigh just above the knee of the human body. The receiving node was attached to the chest at all times (shown in Fig. 2).

Throughout the tasks, body movement acceleration data with a sampling rate of $100 \mathrm{~Hz}$ were logged and then sent from the transmitting node, changing the transmitter location around the legs and arms of the human body allow link comparisons to be made for different node positions. The data were also logged by the receiving node located at the chest. The transmitted and received packets were compared to calculate the transmission reliability as a percentage. The sample rate is programmable and can be set differently for different applications. A low sample rate of $(10 \mathrm{~Hz})$ would not give a clear view of the human movement (as shown Fig.3) and therefore is not the best choice. A larger sample rate (such as $200 \mathrm{~Hz}$ ) will give more detail than required and consequently will consume more power from both the transmitter and receiver as well as channel capacity and memory.
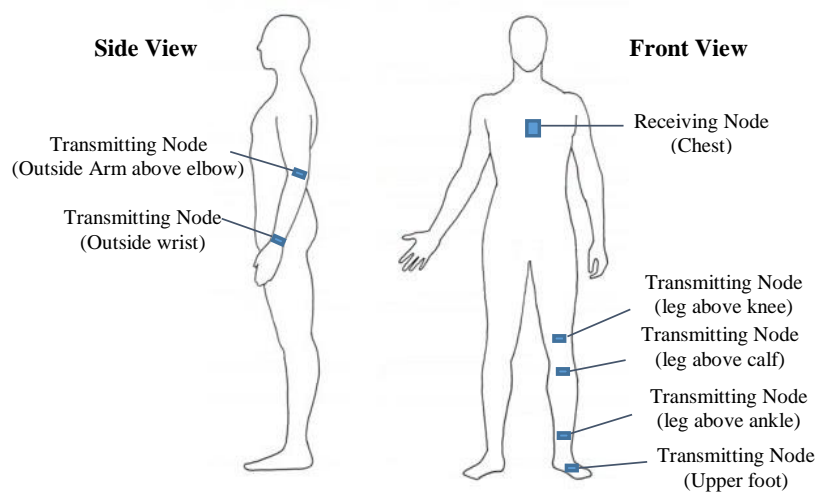

Fig. 2. Body locations for the wireless sensor nodes. (a)

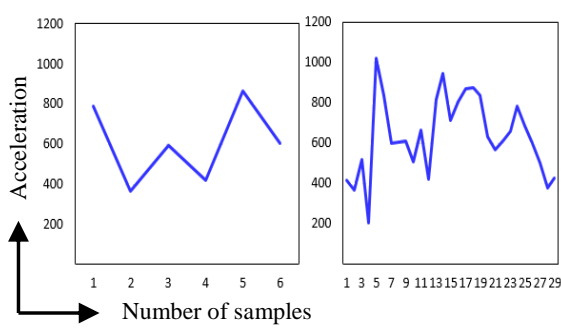

(c)

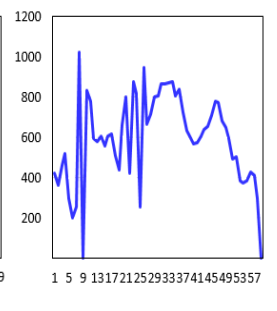

Fig. 3. The effect of different sample rates for one swing (a) $10 \mathrm{~Hz}$ (b) $100 \mathrm{~Hz}$ (c) $200 \mathrm{~Hz}$ demonstrate the effect of under-sampling.

Therefore, $100 \mathrm{~Hz}$ sampling rate was used as an optimized choice to fit the needs of the study.

\section{EXPERIMENTAL RESULTS}

Several types of different tests were conducted using the system described in Fig. 1 and 2.

\section{A. Free space test (Line of Sight)}

Data rate measurements were undertaken in free space in order to characterize the wireless link without a human body. In the test, a distance of 2 meters (more than the normal human height) was set between the transmitter and receiver nodes which were configured at same power level and frequency $[0 \mathrm{dBm}$ and $2.4 \mathrm{GHz}]$. The test showed that no data was lost for channel data transmission rates of $250 \mathrm{kbps}, 1 \mathrm{Mbps}$ and $2 \mathrm{Mbps}$.

\section{B. Human based test}

Three experiments were performed to investigate the effect of body movements, sensor locations and wireless setting on the successful transmission rate during running. All of the tests took place in a sports field. The participants were asked to run a distance of 20 meters at their moderate speed starting from a stationary position and ending at a stationary position; this distance is sufficient for the runner to reach that speed. The swing speed was calculated instead of the running speed to find a direct relationship between the speed of the limb and the link reliability. Further, it is known that the swing speed varies little with running speed [7]. 
1) Different participants, One sensor location and Data rate: The wireless nodes were installed on the arm and chest of five different participants with fixed data rate of $2 \mathrm{Mbps}$. Table I shows the running experience, age, height and weight of the participants and the successful data transmission rate during running. The table also shows the average swing time calculations of the five participants when running at uniform speed. The swing time measurement was made using an algorithm developed in MatLab. The algorithm checks all minimum values which have acceleration value that is smaller than the sample before and after it. This checker ensures that only one value is selected when there are multiple minimum values. Then the lowest value among all recorded minimum values in one sample window is selected and a pointer holds its location. The same process is repeated for another sample window to calculate the time difference between the first and second pointers of the lowest values which equals to the swing time. Fig. 4 describes the swing time calculation for different runners. The sample window can be set manually for each runner or it can be defined by using another calibration algorithem.

The differences in swing time are drived by the participants running style, running experience and the characteristic limb movement during the action of running. The data losses of the wireless nodes for different runners are stongly correlated (correlation cofficient of $89 \%$ ) with the longest or shortest swing time a runner can have.

Fig. 5 shows the recorded data of participant $A$ at the arm location after applying a swing time calculation algorithm, the shadowed area represent the starting and stopping time required by the participant to get into a moderate running speed at constant velocity. These shadowed areas were not included in the calculation of average swing time of participants shown in Table I. Also it was not included in the calculation of successful received acceleration sample rate shown in Fig. 6, since the swing time and sensor location will be different to that recorded when the participant is running at constant speed.

2) One participant, Different sensor locations and Data rate: Participant A was asked to wear the wireless sensors in six different locations for a channel data rate setting of $250 \mathrm{kbps}, 1 \mathrm{Mbps}$ and $2 \mathrm{Mbps}$, respectively. The eighteen recorded sessions from the wireless transmitter and receiver nodes revealed the data percentage received in each sensor location and for each data rate value used during sprinting.

Fig. 6 shows the acceleration data transmitted and received for each wireless node location and data rate value during running. These recorded data are acceleration measurements for a rhythmic running action for all sensor locations. The received data percentages are directly related to the channel data rate and the highest data loss occurred when a data rate of 2Mbps was used.
TABLE I

Participant pool and successful transmission rate.

\begin{tabular}{ccccccc}
\hline \hline $\begin{array}{c}\text { Participant } \\
\text { ID }\end{array}$ & $\begin{array}{c}\text { Running } \\
\text { training } \\
\text { (hour/week) }\end{array}$ & $\begin{array}{c}\text { Age } \\
\text { (years) }\end{array}$ & $\begin{array}{c}\text { Height } \\
(\mathrm{m})\end{array}$ & $\begin{array}{c}\text { Weight } \\
(\mathrm{kg})\end{array}$ & $\begin{array}{c}\text { Swing } \\
\text { Time } \\
(\mathrm{ms})\end{array}$ & $\begin{array}{c}\text { Success } \\
\text { Rate }\end{array}$ \\
\hline A & 1 & 28 & 1.75 & 85 & 510 & $54 \%$ \\
B & 0 & 32 & 1.78 & 68 & 490 & $56 \%$ \\
C & 1 & 22 & 1.7 & 70 & 400 & $85 \%$ \\
D & 2 & 22 & 1.8 & 72 & 420 & $95 \%$ \\
E & 1 & 23 & 1.79 & 69 & 400 & $98 \%$ \\
\hline \hline
\end{tabular}

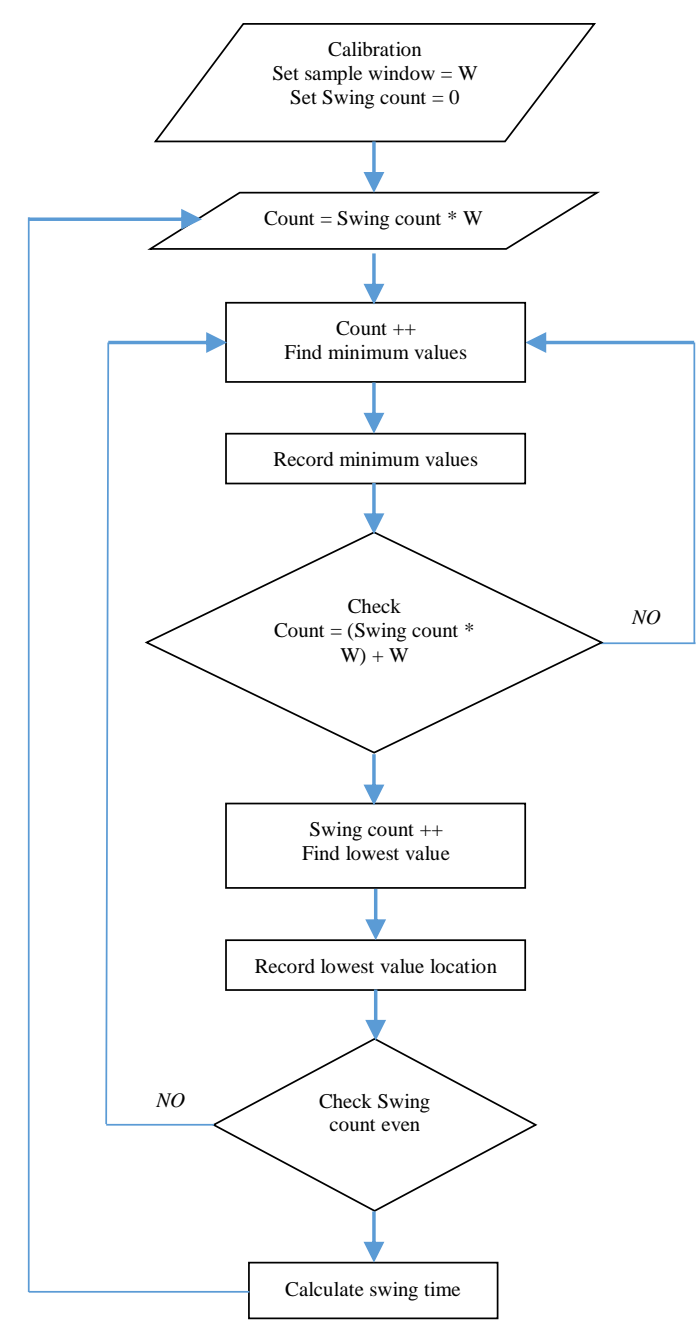

Fig. 4. Flowchart describes the swing time calculation algorithm.

The swing speed at each sensor location was determined by applying the swing time algorithm. Using the algorithm, checking for the maximum instead of the minimum values gives more accurate swing time calculations for acceleration signals with more categorical peak patterns. 


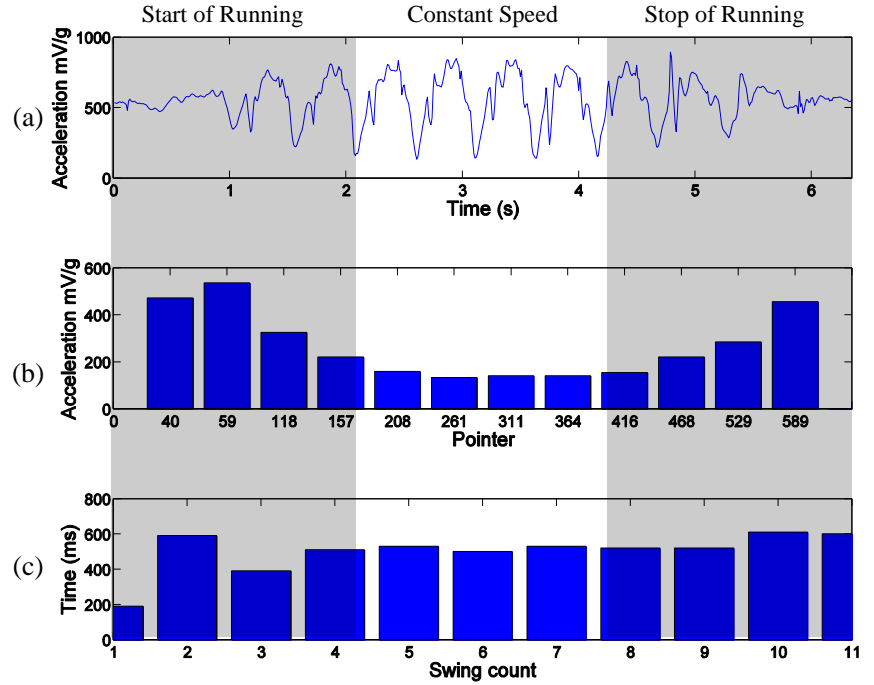

Fig. 5. Participant A (a) acceleration data at arm location (b) lowest values in the sample window (c) swing time calculated values.
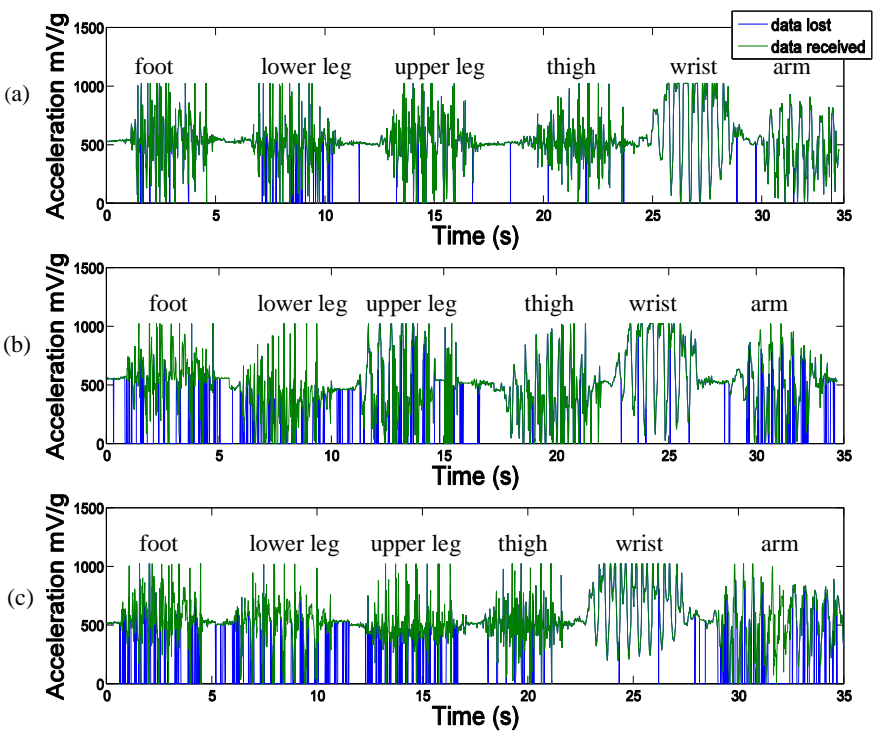

Fig. 6. Transmitted (Blue) and received (Green) data during running for multiple body locations using a data rate of (a) 250kbps (b) $1 \mathrm{Mbps}$ (c) $2 \mathrm{Mbps}$. The same sensor was placed on the foot, lower leg, upper leg, thigh, wrist and arm sequentially while the participant ran for $20 \mathrm{~m}$ starting and ending at a stationary position.

Fig. 7 shows the link reliability for each sensor location and the data rate value used. The lower the data rate the higher the link reliability, as each data rate requires different receiver sensitivity $(-82 \mathrm{dBm}$ for $2 \mathrm{Mbps},-85 \mathrm{dBm}$ for $1 \mathrm{Mbps}$ and $-94 \mathrm{dBm}$ for $250 \mathrm{kbps}$ ), the wrist provides the most consistent results over the data rate changes with a reliability of $98 \%$. This is obviously because the sensor on the wrist during movement continuously provides a received signal level above the lowest sensitivity threshold for each different data rate. The lower leg location gives reliabilities of $25 \%, 42 \%$ and $94 \%$ for data rates of $2 \mathrm{Mbps}, 1 \mathrm{Mbps}$ and $250 \mathrm{kbps}$, respectively.

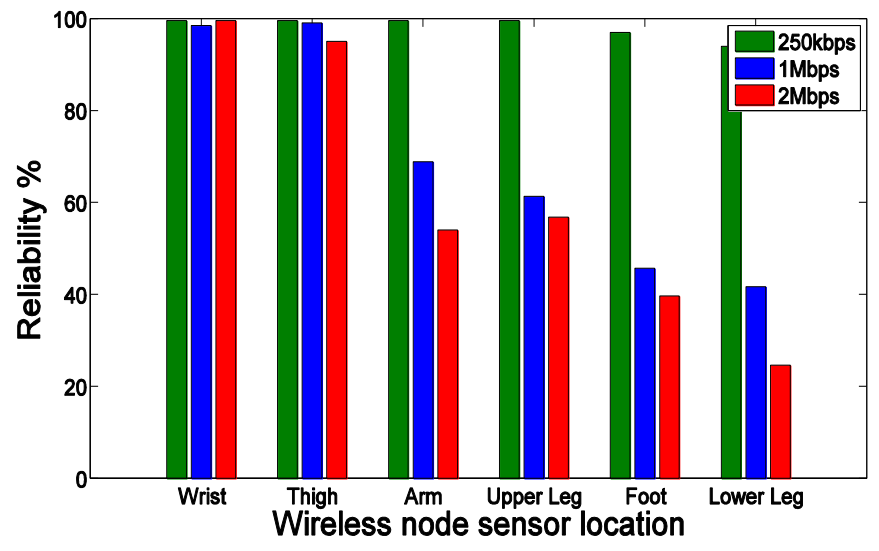

Fig. 7. Link reliability for all sensor locations and three data rates for participant A.

TABLE II

Aggregate percentage of the success rate of participant $\mathrm{A}$ for data rates of $250 \mathrm{kbps}, 1 \mathrm{Mbps}$ and $2 \mathrm{Mbps}$.

\begin{tabular}{cc}
\hline \hline Data Rate & Aggregate successfully received sample rate \% \\
\hline $250 \mathrm{kbps}$ & 98 \\
$1 \mathrm{Mbps}$ & 70 \\
$2 \mathrm{Mbps}$ & 62 \\
\hline \hline
\end{tabular}

Table II gives a general idea of the wireless body area network performance with respect to data rate. The network highest success rate of $98 \%$ resulted using a channel data rate of $250 \mathrm{kbps}$ compared with channel rates of 1 and $2 \mathrm{Mbps}$ which produced a success rate of $70 \%$ and $62 \%$, respectively

3) One Participant, Sensor location and Data rate: The auto acknowledgement feature was enabled to monitor the enhancement of the network success rate. The auto acknowledgement protocol enables the transmitter to wait for the receiver acknowledgement to know whether the packets were received successfully. The acknowledgment packet handling is as follows [11]:

- The transmitter starts the operation by sending a data packet to the receiver. The auto acknowledgement protocol automatically switches the transmitter to receive mode to wait for the ACK packet;

- If the packet is received by the receiver, the receiver auto acknowledgement protocol automatically assembles and transmits the ACK packet to the transmitter before returning to receive mode;

- If the transmitter does not receive the ACK packet; the auto acknowledgment protocol retransmits the original data packet after a programmable delay and sets the transmitter to receive mode to wait for the ACK packet; 
- The process continues for a pre-programmed number of retransmissions until successful delivery or the maximum number of retransmissions was reached;

- If the transmitter does not receive the ACK packet and after reaching the maximum number of retransmission attempts, the data is discarded and the transmitter starts the transmission of a new data packet.

Different maximum numbers of retransmission attempts were programmed into the system and tested with a dynamic sampling rate that allows 100 samples per second. This was performed by differentiating the time delay of retransmissions from the sampling rate at each packet transmission (i.e., acceleration samples are sent every $10 \mathrm{~ms}$, even if there was retransmissions involved for the same sample). The test was conducted on participant $\mathrm{A}$ at the arm position with $1 \mathrm{Mbps}$ data rate.

Fig. 8 shows the total number of packets transmitted through the link and successfully received sample rate during constant running speed of participant $\mathrm{A}$ and for two seconds. The success rate significantly rises from $69 \%$ to $88 \%$ with an increasing number of retransmissions. However, the retransmissions increases the traffic load on the wireless link and a success rate of nearly $69 \%$ requires the transmission of 200 packets with no retransmission while a success rate of nearly $88 \%$ requires more than four times that number.

Table III provides an indication of the traffic generation for different retransmission settings. For the 200 packets the ideal goodput is $2.4 \mathrm{Kbps}$ (knowing that each sample payload is 24 bits long and the sampling rate is $100 \mathrm{~Hz}$ ). From the table it can be seen that the goodput increases when the number of packet retransmissions is larger. Nevertheless this small improvement required more than 3.4 times the original traffic that was to be delivered in one second. This data loss reduces the link capacity and is not practical for applications where continuous data is sent. This is more of a problem in large networks where more than two wireless nodes are communicating, but it would be valuable for applications in which a single or few transmissions happened in one second and link reliability is important.

In this experiment only one sensor location (on the arm) with one data rate (1Mbps) was chosen, to show clearly the wireless link enhancement for different retransmission attempts. It showed improvement in the link reliability on the account of traffic lost and channel capacity. Changing the sensor location will give the same conclusion as that obtained from the arm location as well as the data rates. Therefore; different running styles, sensor locations, and data rates would have differing effects on the link reliability and would require different retransmission rates. (a)

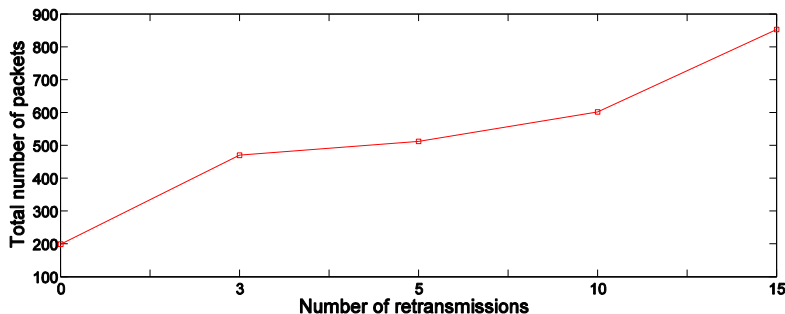

(b)

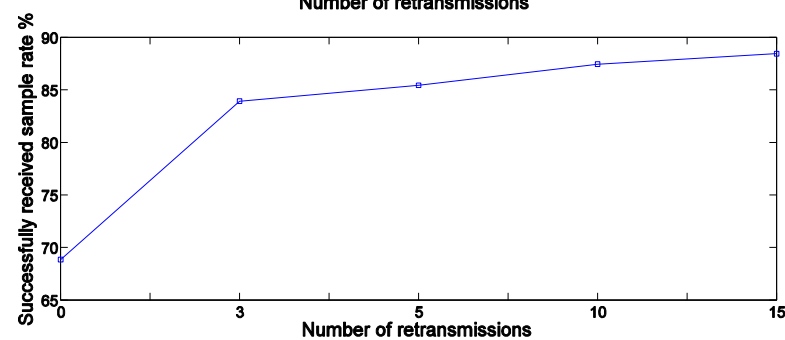

Fig. 8. Number of packets retransmissions with respect to the: (a) total number of packets transmitted and retransmitted (b) successfully received sample rate.

TABLE III

Wireless link performance for different retransmission settings at arm sensor location of participant A.

\begin{tabular}{cccc}
\hline \hline $\begin{array}{c}\text { Maximum } \\
\text { retransmission }\end{array}$ & Total traffic kbps & Goodput kbps & Lost traffic kbps \\
\hline 0 & 2.40 & 1.64 & 0.76 \\
3 & 5.64 & 2.00 & 3.64 \\
5 & 6.14 & 2.04 & 4.10 \\
10 & 7.22 & 2.09 & 5.13 \\
15 & 10.24 & 2.11 & 8.13 \\
\hline \hline
\end{tabular}

\section{CONCLUSION}

Wireless gait analysis is determined by using accelerometer sensors with 100 samples per second. Each sensor records acceleration samples per swing action and sends these samples during the appropriate time window to the central unit which in turn will sends them off the body (locally or to the cloud) for further analysis. The results showed that installing the sensor on the wrist provides the best among all selected locations for reliable communications. It also showed that the swing time and running style of different runners are strongly correlated to the percentage of successful transmissions. This study explored the effect of using the auto acknowledgment feature on the link performance and how it can be implemented for different applications. Future work will involve the design of learning algorithms that automatically enhance the wireless link reliability by changing one or more of these factors for each different sensor position and user.

\section{ACKNOWLEDGEMENT}

This work was supported by the Higher Committee for Education Development in Iraq (HCED) as part of Haider A. Sabti's Ph.D. program. The authors would also like to thank the participants for their participation and Griffith University for granting access to important resources. This research was conducted under Griffith University Ethics approval number ENG/20/13/HREC. 


\section{REFERENCES}

[1] S. Sarkar, P. J. Phillips, Z. Liu, I. R. Vega, P. Grother, and K. W. Bowyer, "The humanID gait challenge problem: data sets, performance, and analysis," IEEE Trans Pattern Anal Mach Intell, vol. 27, pp. 162-77, Feb 2005.

[2] P. S. Hall and Y. Hao, "Antennas and propagation for body centric communications," First European Conference on Antennas and Propagation, pp. 1-7. EuCAP 2006.

[3] L. M. Borges, A. Rente, F. J. Velez, L. R. Salvado, A. S. Lebres, J. M. Oliveira, et al., "Overview of progress in Smart-Clothing project for health monitoring and sport applications," First International Symposium on Applied Sciences on Biomedical and Communication Technologies, pp. 1-6, ISABEL '08, 2008.

[4] H. S. Cao, V. Leung, C. Chow, and H. Chan, "Enabling Technologies for Wireless Body Area Networks: A Survey and Outlook," IEEE Communications Magazine, vol. 47, pp. 84-93, Dec 2009.

[5] J. Zhao and R. Govindan, "Understanding packet delivery performance in dense wireless sensor networks," presented at the Proceedings of the 1st international conference on Embedded networked sensor systems, Los Angeles, California, USA, 2003.

[6] S. L. Cotton and W. G. Scanlon, "A Statistical Analysis of Indoor Multipath Fading for a Narrowband Wireless Body Area Network," IEEE 17th International Symposium on Personal, Indoor and Mobile Radio Communications, pp. 1-5, 2006.

[7] P. G. Weyand, D. B. Sternlight, M. J. Bellizzi, and S. Wright, "Faster top running speeds are achieved with greater ground forces not more rapid leg movements," Journal of Applied Physiology, vol. 89, pp. 1991-1999, November, 2000.

[8] B. Latré, B. Braem, I. Moerman, C. Blondia, and P. Demeester, "A survey on wireless body area networks," Wireless Networks, vol. 17, pp. 1-18, 2011.

[9] H. A. Sabti and D. V. Thiel, "Movement based time division multiplexing for near real time feedback body area network applications," presented at the International Workshop on Antenna Technology, Sydney, iWAT 2014.

[10] M. K. Maggs, S. G. O'Keefe, and D. V. Thiel, "Consensus Clock Synchronization for Wireless Sensor Networks," IEEE Sensors Journal, vol. 12, pp. 2269-2277, Jun 2012.

[11] N. Semiconductor. (2008, 2013). nRF24L01+ Single Chip 2.4GHz Transceiver Preliminary Product Specification V1.0. Available: http://www.nordicsemi.com/eng/Products/2.4GHz-RF/nRF24L01

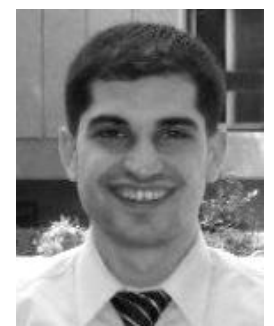

Haider A. Sabti received the B.Sc. degree in electronics and communication engineering from Al-Nahrain University, Baghdad, Iraq, in 2007, and the M.Sc. degree in electronics and communication engineering in 2010. $\mathrm{He}$ is currently pursuing his Ph.D. degree in wireless body area network for running sport applications. $\mathrm{He}$ is an IEEE student member and a member of the Centre for Wireless Monitoring and Applications at Griffith University, Brisbane, Australia. $\mathrm{He}$ has been working as an RF engineer in a CDMA communication company in Baghdad, Iraq, since 2010. He is supported by a scholarship from HCED in Iraq. His current research interests include wearable wireless sensor networks, design and implementation of smart sensors, embedded systems for athletes monitoring, and sport data analysis.

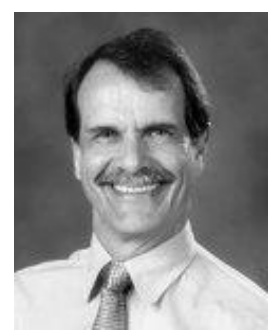

David Victor Thiel received the degree in physics and applied mathematics from the University of Adelaide, Adelaide, Australia, and the Masters and Ph.D. degrees from James Cook University, Townsville, Australia. He is currently the Director of the Centre for Wireless Monitoring and Applications with Griffith University, Nathan, Australia. He has coauthored Switched Parasitic Antennas for Cellular Communications. His interests include mathematical optimization techniques for antenna design. He has published over 100 journal papers, more than 150 papers presented at international conferences, and he has co-authored more than nine patent applications. He was a co-inventor of the new RoHS and WEEE compliant electronics manufacturing technology called "circuits in plastic." His current research interests include electromagnetic geophysics, sensor development, electronics systems design and manufacture, and antenna development for wireless sensor networks. He is a fellow of the Institution of Engineers, Australia. $\mathrm{He}$ is currently the Chair of the IEEE Wave Propagation Standards Committee and serves on the IEEE Antenna Standards Committee. 